\title{
Complications and Effectiveness of Intramedullary Limb Lengthening: A Matched Pair Analysis of Two Different Lengthening Nails
}

\author{
Peter H Thaller ${ }^{1}$, Felix Frankenberg ${ }^{2}$, Nikolaus Degen ${ }^{3}$, Chris Soo ${ }^{4}$, Florian Wolf ${ }^{5}$, Ekkehard Euler ${ }^{6}$, Julian Fürmetz ${ }^{7}$
}

\begin{abstract}
Background: Intramedullary limb lengthening has become an accepted concept in reconstructive surgery, but as yet comparative clinical studies are missing. We compared the complications and effectiveness of two types of intramedullary limb lengthening devices (ISKD ${ }^{\circledR}$; Fitbone ${ }^{\circ}$ ).

Materials and methods: In a retrospective series of 278 consecutive patients with internal limb lengthening, we found 17 matching pairs in terms of predefined matching parameters (group I with ISKD ${ }^{\odot}$ and group II with Fitbone ${ }^{\odot}$ ). The surgeries were all performed with the same technique and managed with equivalent pre- and postoperative treatment protocols. The performance of the implants was evaluated using the distraction index and the weight-bearing index. Complications were rated according to Paley's classification for external lengthening.

Results: The distraction index in group I (ISKD ${ }^{\oplus}$ ) was $0.99 \mathrm{~mm} /$ day (range $0.55-1.67$ ) and in the group II (Fitbone ${ }^{\circ}$ ) $0.55 \mathrm{~mm} /$ day (range 0.14-0.92) ( $p$ value $=0.001$ ). The mean weight-bearing index differed between group I and group II from 32.0 day $/ \mathrm{cm}$ (range 16.4-64.0) to $51.6 \mathrm{day} / \mathrm{cm}$ $(25.8-95.0)(p$ value $=0.001)$. There were 17 recorded incidents in group I and 19 in group II during lengthening .

Conclusion: Specific technical handicaps of the two systems, such as the so-called runaway of the ISKD ${ }^{\circledR}$ and backtracking of the Fitbone ${ }^{\bullet}$ nails seem to result in different distraction index and weight-bearing index. Further comparative studies might induce technical progress in intramedullary limb lengthening.

Keywords: Distraction osteogenesis, Fitbone ${ }^{\oplus}$, Intramedullary lengthening nails, ISKD ${ }^{\oplus}$, Limb lengthening.

Strategies in Trauma and Limb Reconstruction (2020): 10.5005/jp-journals-10080-1448
\end{abstract}

\section{INTRODUCTION}

Intramedullary lengthening nails have several advantages in the management of leg length discrepancies such as lower infection rates, less soft tissue damage and pain, better joint movement, better alignment control, and more patient comfort compared to external devices. ${ }^{1-6}$ With the rising number of intramedullary lengthening procedures over the past years, six different fully implantable intramedullary nails have been investigated in the literature, including the Bliskunov-Nail, the Albizzia-Nail, and the Phenix ${ }^{\oplus}$ nail, which are no longer available; and the Intramedullary Skeletal Kinetic Distractor (ISKD ${ }^{\circledR}$, Orthofix, McKinney), which is now available again in Europe. ${ }^{3,5,7,8}$ Currently, the Fitbone ${ }^{\circledast}$ nail (Wittenstein Intens1, Igersheim, Germany) and the Precice ${ }^{\oplus}$ nail (Ellipse Technologies Inc., CA, USA) are the most frequently implanted systems worldwide. .,9-12 $^{6}$

These nails are characterised by different actuation mechanisms, and each have their specific limitations. The Phenix ${ }^{\circledR}$ nail and the Precice $^{\oplus}$ nails are magneto-mechanically driven systems; the Bliskunov-nail, the Albizzia-nail, and the ISKD ${ }^{\circledR}$ are mechanically driven; and the Fitbone ${ }^{\circledR}$ nail is a motorised (electromotive) system. ${ }^{2,3,6-8,11,13}$

In the literature, the main problems of intramedullary lengthening devices mentioned are control of distraction rate, maintenance of distraction and implant failure. . $^{8,9,14-16}$

The Fitbone ${ }^{\circledR}$ nail was developed at our clinic in $1990 .{ }^{1}$ In 2006, we reported on 150 lengthening procedures with the Fitbone ${ }^{\oplus}$ nail. ${ }^{17}$ A total of $144(96 \%)$ patients reached the distraction goal, there was no infection, implant failure occurred in 3 cases, and 9 other instances of technical difficulty were reported. The unintended
1,3,5-73DSurgery, Department of General, Trauma and Reconstructive Surgery, Munich University Hospital, LMU, Germany

${ }^{2}$ Department Trauma and Reconstructive Surgery, Klinikum München Süd, Germany

${ }^{4}$ School of Medicine, Griffith University, Gold Coast, Australia

Corresponding Author: Julian Fürmetz, 3DSurgery, Department of General, Trauma and Reconstructive Surgery, Munich University Hospital, LMU, Germany, Phone: +49 894400 55401, e-mail: Julian. Fuermetz@med.uni-muenchen.de

How to cite this article: Thaller PH, Frankenberg F, Degen $\mathrm{N}$, et al. Complications and Effectiveness of Intramedullary Limb Lengthening: A Matched Pair Analysis of Two Different Lengthening Nails. Strategies Trauma Limb Reconstr 2020;15(1):7-12.

Source of support: Nil

Conflict of interest: None

back tracking of the implant as described in this early paper was detected in nine $(6 \%)$ cases.

In 2009, we gathered first-hand experience with the mechanically driven ISKD ${ }^{\circledR}$ nail that was first described by Dean Cole in 2001. ${ }^{2}$ At the time it was the most implanted system and by far the most published one. In our initial comparison of our early results to the Fitbone ${ }^{\circledR}$ nail, we also observed a few handicaps of ISKD ${ }^{\circledR}$ nail. ${ }^{18}$ It was withdrawn from the US market, temporarily in 2009 and finally in 2012. The main reasons were jammed or nondistracting nails, leading to early consolidation, ${ }^{14}$ and unintended high distraction rates ("runaway" or "accelerated" nail). ${ }^{15}$

(0) The Author(s). 2020 Open Access This article is distributed under the terms of the Creative Commons Attribution 4.0 International License (https://creativecommons. org/licenses/by-nc/4.0/), which permits unrestricted use, distribution, and non-commercial reproduction in any medium, provided you give appropriate credit to the original author(s) and the source, provide a link to the Creative Commons license, and indicate if changes were made. The Creative Commons Public Domain Dedication waiver (http://creativecommons.org/publicdomain/zero/1.0/) applies to the data made available in this article, unless otherwise stated. 
A scientific evaluation between intramedullary lengthening nails is difficult, as the number of implantations is low worldwide and techniques vary significantly between surgeons.

By now, we were able to gather experience with five different intramedullary lengthening devices. ${ }^{13}$ On those and other intramedullary lengthening nails, there are multiple publications of specific case series. ${ }^{1-3,8,11,14}$ There are also studies comparing intramedullary lengthening with lengthening over nail (LON) or with LRS external fixator monorail system. ${ }^{19,20}$ We found two matched-pair studies on LON vs external lengthening (llizarov) and one on intramedullary lengthening (Fitbone ${ }^{\circledR}$ ) vs external lengthening (Taylor Spatial Frame). ${ }^{21-23}$ But there are no matched pairs studies that compare specific intramedullary lengthening devices.

This is the first matched pair analysis comparing two different intramedullary lengthening devices (ISKD ${ }^{\oplus}$ and Fitbone ${ }^{\oplus}$ ).

\section{Materials and Methods}

In a retrospective review of a consecutive series, we found 278 patients who had lengthening of the lower limb between March 1999 and November 2011. The study was approved by our Institutional Review Board. From March 1999 to June 2009, we performed 241 limb lengthening procedures with a fully implantable electromechanicalactuated intramedullary nail (Fitbone ${ }^{\oplus}$ ). From July 2009 to November 2011, 37 consecutive limb lengthening procedures were performed using a fully implantable mechanical-actuated intramedullary nail (ISKD ${ }^{\circledR}$ ). All procedures were conducted by the same surgeon with an equivalent technique (see below).

\section{Matching Criteria}

The case matching was done as per agreement in five criteria:

- Equivalent surgical technique and pre- and postoperative treatment protocol

- Site of osteotomy (proximal femur, distal femur or proximal tibia)

- Simultaneous realignment of the mechanical axis (yes or no)

- Amount of lengthening (maximum variation 10\%)

- Age (maximum variation $20 \%$ ).

All five criteria had to comply with both matching partners. Subsequent lengthening of another bone, implant failure due to malpractice by the patient or accidental trauma, or nicotine abuse during lengthening led to exclusion. Mismatch in sex and aetiology of leg length discrepancy were not included in the matching criteria.

\section{Outcome Measures}

Primary outcome measures included completed lengthening, distraction index (DI) in $\mathrm{mm} /$ day, and weight bearing index (WBI) in day/cm, as described in former publications. $4,5,8,9,12,15,24$

We classified problems, obstacles, and complications as they are described for external lengthening by Paley in $1990 .{ }^{25}$ Implantrelated complications were specified as recommended by Lee et al. ${ }^{26}$

\section{Surgical Technique and Treatment Protocol}

Preoperative analysis, end-point-first planning, and operative technique with protective sleeves, rigid reaming, X-ray-grid verification were the same in all procedures. We have previously published detailed descriptions of our operative technique for the tibia and femur. ${ }^{27-29}$
Postoperatively, patients received calcium and vitamin D, lowmolecular-weight heparin and analgesics according to the WHO scheme (with the exception of NSAIDs). Prophylactic antibiotic treatment was maintained for as long as drains were in situ, in most cases for 1 day. All patients had regular physical therapy and were mobilised with partial weight bearing of $20 \mathrm{~kg}$ from the first day on. All patients were instructed to conduct lengthening procedures autonomously.

The distraction process started on the fifth postoperative day and was controlled radiologically at least twice prior the patient's discharge to confirm that the distraction gap is opening. After discharge, clinical examination and radiological control were performed every week. In the majority of cases, the intended DI was $1 \mathrm{~mm}$ /day in all patients, with $0.33 \mathrm{~mm}$ lengthening applied three times daily. In very few cases the distraction speed was adjusted according to radiological signs of consolidation of the bony regenerate, conditions of joints and neurovascular structures. The range of motion of adjacent joints was controlled at each patient visit. After reaching the distraction goal, consolidation was monitored initially every 2 weeks and after partial consolidation every 6 weeks.

As soon as one cortical side of the regenerate was deemed radiologically stable, weight bearing was increased gradually until full weight bearing was achieved within 6 weeks at the latest. The WI was calculated by the start of full weight bearing. Full consolidation and removal of the implants were achieved after 12-18 months. Six months after removal, a final reassessment of clinical and radiological parameters, including the leg length and alignment, was performed (Fig. 1).

\section{Statistics}

All matched variables were evaluated for statistical significance between groups using a Wilcoxon test for paired variables (SPSS 23). $p$ values of less than 0.05 were regarded as statistically significant.

\section{IRB approval}

The study was carried out according to the Declaration of Helsinki. The Ethic Committee of the University of Munich approved this study with the ID number 8-16.

\section{Results}

From 37 ISKD ${ }^{\circledR}$ patients, we excluded two patients due to a simultaneous lengthening of the tibia or femur and two patients due to nicotine abuse during lengthening.

From 33 remaining ISKD ${ }^{\circledR}$ patients, 17 patients (group I) could be matched to 17 Fitbone ${ }^{\circledast}$ patients (group II) respecting all five of the aforementioned criteria. The pairs of lengthening procedures were 4 proximal femoral, 7 distal femoral, and 6 proximal tibial (Table 1).

Mean follow-up of all selected patients was 2.5 years (range 1.3-7.0 years). All 34 patients completed the lengthening process. The average DI for ISKD ${ }^{\circledR}$ patients was $0.99 \mathrm{~mm} /$ day (range $0.55-1.67$ $\mathrm{mm} /$ day) and for Fitbone ${ }^{\circledast}$ patients $0.55 \mathrm{~mm}$ /day (range $0.14-0.92$ $\mathrm{mm} /$ day). The difference in DI between group I and II was significant $(p=0.001$ ). Mean WBI was $32.0 \mathrm{day} / \mathrm{cm}$ (range 16.4-64.0 day/cm) in the ISKD group and $51.6 \mathrm{day} / \mathrm{cm}$ (range $25.8-95.0 \mathrm{day} / \mathrm{cm}$ ) in the Fitbone ${ }^{\circledast}$ group. The WBI between group I and II was significantly different $(p=0.001)$. Differences between the three osteotomy sites independent from group I or II were not significant $(p=0.875)$.

Detailed patient data and results are shown in Table 1. 

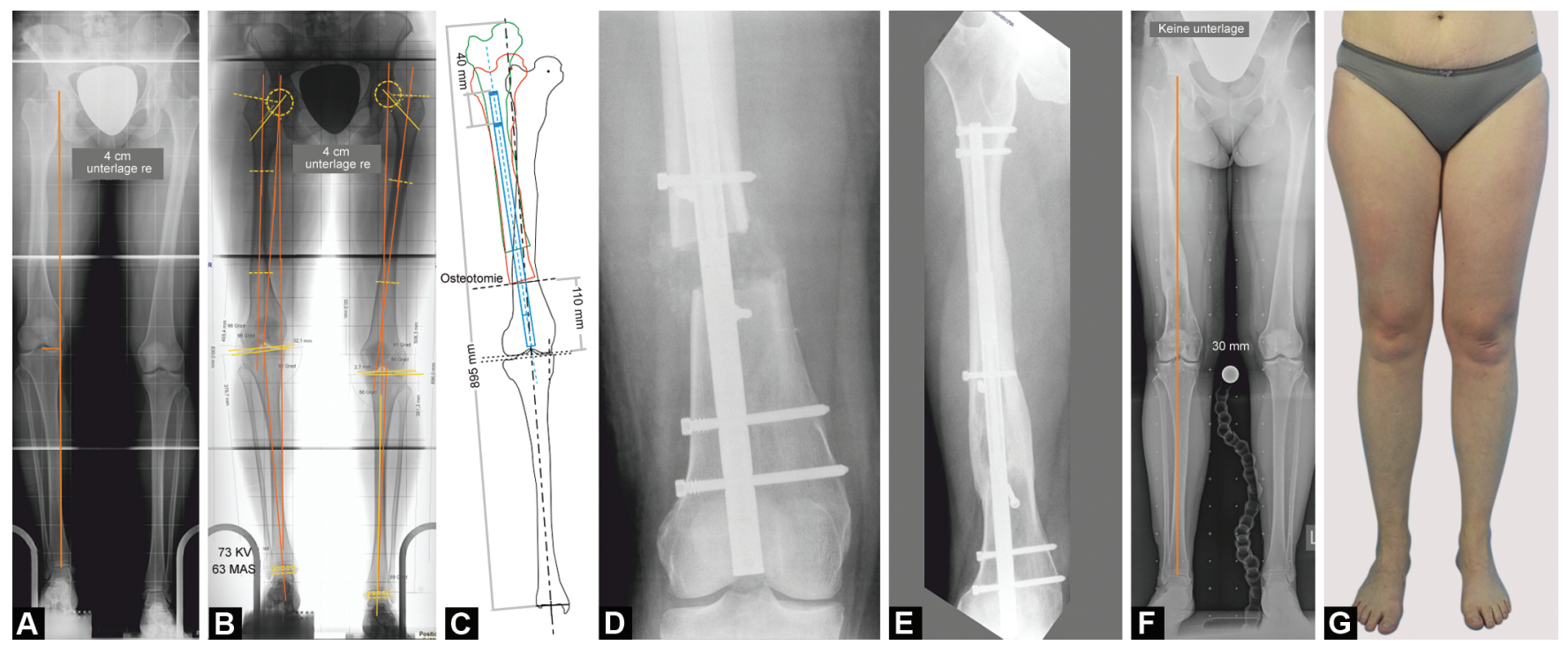

Figs 1 A to G: (A) Preoperative long-standing radiograph with post-traumatic shortening with varus deformity of the right femur; (B) Preoperative analysis; (C) End-point-first (EPF) planning for simultaneous lengthening and axis correction with retrograde lengthening nail (ISKD); (D) Ongoing lengthening, the blocking screw in the metaphyseal fragment stabilises the nail for the necessary valgisation, in the diaphyseal part, the medial and dorsal blocking screw enhances the friction at the rotating ISKD shaft for a better control of lengthening; (E) Complete lengthening achieved; (F) Long-standing radiograph 6 months after implant removal; (G) Final clinical result, follow-up 4.5 years after lengthening

Patient I-7 had had a long history of previous surgeries with a wider cavity. She additionally suffered from severe agitation and excessive postoperative movement due to drug addiction and unexpected withdrawal symptoms after the surgery. The acute motor agitation triggered accidental distractions in the wider cavity. In an attempt to slow down lengthening, three blocking screws were implanted in an attempt to increase friction between the nail and cortical bone. Additionally, an epidural catheter permitted intermediate motor paresis. In this elderly patient, insufficient callus formation was managed by an exchange to a straightened conventional intramedullary nail and bone grafting after 6 months, with no further problems and full consolidation.

\section{Complications}

Regarding Paley's classification for external lengthening, we noted no complication in either of the groups.

In the ISKD ${ }^{\circledR}$ group (I), we observed 11 problems in 7 patients and 6 obstacles in 4 patients. Problems in group I: $3 \times$ pain treated in an inpatient setting with peridural catheter or other analgesics, $1 \times$ accelerated nail treated with peridural catheter to reduce patient movements, $2 \times$ equinus treated with physiotherapy successfully, $1 \times$ broken interlocking bolt, $2 \times$ temporary peroneal nerve irritation, $1 \times$ superficial wound defect, and $1 \times$ thrombosis of the popliteal vein. Obstacles in group I: There were two cases of early consolidation treated by re-osteotomy, which did not affect the final outcome. Aforementioned accelerated nail treated with peridural catheter received additionally three blocking screws to increase the friction.

In the Fitbone ${ }^{\oplus}$ group (II) there were 13 problems in 11 patients and 6 obstacles in 5 patients. Problems in group II: $1 \times$ defect in external controller, $7 \times$ back tracking of the nail (DI below $0.5 \mathrm{~mm} /$ day), $3 \times$ temporary peroneal nerve irritation, $1 \times$ periostitis, $1 \times$ equinus foot. Obstacles in group II: $3 \times$ loosening of interlocking bolts (retightening or exchange), $2 \times$ insufficient regenerate by following cancellous bone grafting, $1 \times$ osteophyte requiring removal.
There were neither breakage nor deep infection in either of the groups.

But using Lee's classification for complications with internal lengthening devices we noted 15 implant related complications. ${ }^{26}$ There were four implant related complications with ISKD ${ }^{\oplus}: 3 x$ type I distraction control related ( $1 \times$ accelerated nail, $2 \times$ early consolidations), $1 \times$ type II stability related (broken interlocking bolt). Additionally, there were 11 implant-related complications with Fitbone ${ }^{\circledast}$ : $8 \times$ type I distraction control related (1X defect controller, $7 \times$ back tracking), $3 \times$ type II stability related (loosening of interlocking bolts).

No implant related complication affected the final outcome.

\section{Discussion}

Until now, there are only case series on individual implants to evaluate treatment efficacy and complications of intramedullary limb lengthening devices. This is the first comparison of two different implants (ISKD ${ }^{\circledast}$ and Fitbone ${ }^{\oplus}$ ) in a matched pair analysis. Our results show a similar rate of problems, obstacles, and complications but indicate significant differences in the lengthening and consolidation phase (DI and $\mathrm{WBI}$ ) between the two devices.

A problem frequently reported with the ISKD ${ }^{\circledR}$ is the insufficient control of distraction speed. An unintentionally distracting nail with a DI of more than $1.5 \mathrm{~mm} /$ day was named as an "accelerated nail" or "runaway nail". From 2001 to 2012, the ISKD ${ }^{\circledR}$ nail was the most implanted intramedullary lengthening device. The earliest ISKD $^{\circledR}$ complication, a $10 \%$ implant failure rate, was reported by Cole et al., with further studies demonstrating a significant risk of early consolidation resulting from non-distracting ISKD ${ }^{\oplus} \mathrm{s}^{2}$ More recently, excessive distraction by runaway nails has been reported, with resultant non-union and insufficient bone regeneration. 2,5,30 Runaway and non-distracting ISKD ${ }^{\circledR}$ incidence have been reported to be up to $50 \% .{ }^{30}$ In 2011, Burghardt et al. published the largest retrospective study about 242 ISKD ${ }^{\otimes}$ lengthening procedures. ${ }^{14} 15$ $I S K D^{\oplus} \mathrm{S}(6.2 \%)$ failed mechanically, with 10 cases of device fracture. 


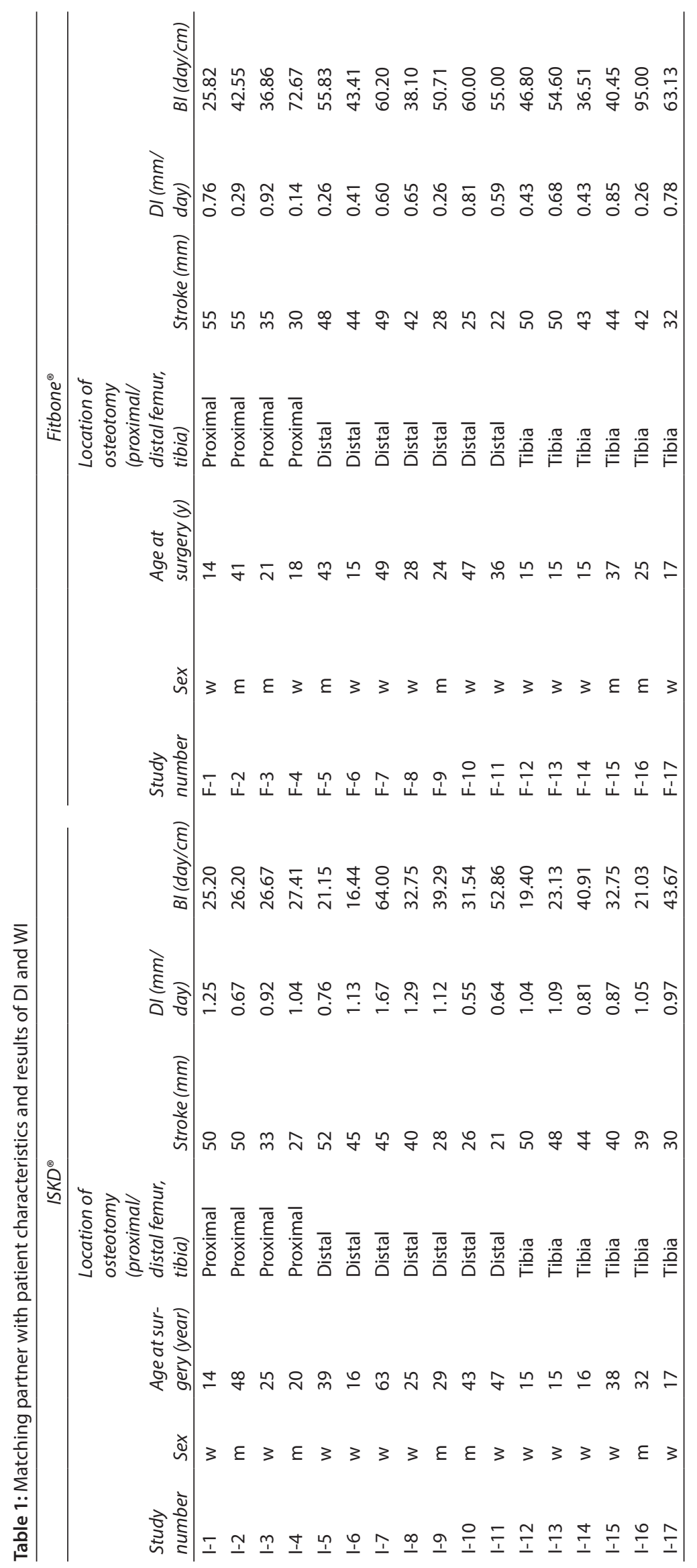


After a further 4 devices reportedly failed to lengthen, thought to be due to an error in the manufacturing process, a worldwide recall of the implant was enacted. However, no cases of "runaway nail" were reported.

In 2006, we reported on 297 Fitbone $^{\circledR}$ applications worldwide. 181 implantations were performed at our university hospital and 150 cases were retrospectively evaluated. A fracture of the nail occurred in $2 \%$ of the cases and unintended back tracking in $5 \% .{ }^{17}$ Fitbone ${ }^{\oplus}$ lengthening devices are not widely available; they are restricted to few clinical centres worldwide, and with the formation of a new group we introduced the ISKD in 2009.

Krieg et al. observed three (9.4\%) cases of backtracking in the consolidation phase of 32 Fitbone $^{\oplus}$ applications. ${ }^{12}$ Backtracking seems to be caused by thousands of repetitive micro-movements at the telescoping part of the nail. These micro-movements are transmitted through the gearbox to the electric motor and cause shortening until the bone is consolidated. In a recently published study of 23 Fitbone ${ }^{\circledast}$ implants, no backtracking was reported and thought to be attributed to a minor technical improvement made by the manufacturer intended to prevent backtracking of the system. ${ }^{31}$ Future independent studies with a larger sample size might further determine if this problem has been solved.

In a number of Fitbone ${ }^{\circledast}$ implantations, we observed signs of nail corrosion at the site, where the telescoping part leaves the main body of the nail. In some cases, we observed osteolysis at this site, and in very few cases, a painful tumorigenic reaction which resolved immediately after removal. ${ }^{18}$ But this was not observed in the patients included in this matched pairs study.

For the ISKD ${ }^{\oplus}$, the $\mathrm{DI}$ is reported to be from 1.1 to $1.5 \mathrm{~mm}$ /day for the femur and 0.5 to $0.9 \mathrm{~mm} /$ day for the tibia. ${ }^{5,15,24,32}$ Case series with the Fitbone ${ }^{\circledast}$ demonstrate a DI from 1.0 to $1.1 \mathrm{~mm} /$ day. $^{10,33}$ In literature, the WBI ranges from 23.3 to 47.8 days/cm for ISKD ${ }^{\circledR}$ and 30.5 to 35.0 days/cm for the Fitbone ${ }^{\oplus}{ }^{10,30,32,33}$ The WBI is not reported in every case series with intramedullary limb lengthening and is often described as the healing index. Historically, the terms healing index, consolidation index and WBI are related to the type of distraction in regard to external vs internal implants. Some authors mention a consolidation index rather than the WBI for fully implantable nails or lengthening over nail techniques.

When reviewing the literature on Fitbone or ISKD ${ }^{\circledR}$ devices, our findings were somewhat unexpected. In the ISKD ${ }^{\circledR}$ group, we could find no incidences of distraction failure, and there were seven cases of backtracking in the Fitbone ${ }^{\circledR}$ group, representing $41 \%$ of cases.

In our results, only one ISKD ${ }^{\circledR}$ patient had a DI of more than $1.5 \mathrm{~mm}$ /day (patient I-7). Our hypothesis is that none of our cases had been over reamed more than $0.5 \mathrm{~mm}$ for implantation of "straight" lengthening nails. Since the medullary cavities of the lower extremity are anatomically curved (femur to anterior and tibia to posterior), flexible reaming results in excessive over-reaming in an attempt to implant the straight (non-curved) device.

There were no cases of accelerated lengthening in the Fitbone ${ }^{\circledR}$ group. Unintended lengthening rarely seems possible with the Fitbone $^{\circledast}$ technique. Frequent backtracking of the Fitbone ${ }^{\circledR}$ nail resulted in a range of DI from 0.14 to $0.9 \mathrm{~mm} /$ day. In the case of the patient with a DI of $0.14 \mathrm{~mm} /$ day, backtracking had already occurred twice during the distraction phase. In order to prevent loss of lengthening during the consolidation of the regenerate, a continuous distraction was necessary even after reaching the targeted length. In the other cases of backtracking, only one had a DI of more than $0.5 \mathrm{~mm} /$ day. We hypothesize that perhaps the design of the Fitbone ${ }^{\circledR}$, with no intrinsic mechanical mechanism for preventing backtracking could be the reason for this.

\section{Limitations}

The main limitation of the study is a low number of matched pairs, due to challenging matching criteria and the lower caseload in the ISKD ${ }^{\circledast}$ group. We chose the matching criteria like this: (1) Because there are significantly diverging techniques, protocols, and a certain learning curve, for consistency, all patients had to be operated by the same surgeon using the same protocol. (2) Bone formation cannot easily be compared between different osteotomy sites, as it depends on the available blood supply at each location. ${ }^{34}$ (3) Simultaneous realignment was matched because of this risk of impaired bone formation by more offset and/or wider open wedges between fragments. (4) The amount of lengthening has a huge influence on the entire treatment, and so we accepted a variance not more than $10 \% .{ }^{22}(5)$ The osteogenic potential decreases over the span of a lifetime, ${ }^{35}$ therefore we matched patients with a maximum variance of $20 \%$ in age. But aetiology was not included in the matching criteria, which can be an important factor for example in cases of CFD (congenital femoral deficiency) with poorer callus formation or Ollier's disease with quicker formation.

There are several other factors which might influence the results of limb lengthening procedures like the conditions of soft tissues, aetiology of the deformity, bone mineral density, and others. Still, we choose only the above-mentioned major matching criteria to prevent an even smaller number of matching partners. We estimate that with more matching criteria or tighter ranges for the specific criteria the number needed to treat would grow higher than any institution can provide.

Another important limitation is that one of the implants has been withdrawn from the market, and the other is not freely available. Nevertheless, we consider the data to be relevant with regard to other internal lengthening systems and believe that further studies may benefit from the methodology used.

One of the issues raised during the study was the lack of an appropriate classification system for internal lengthening systems to facilitate the evaluation of the systems. Although Lee's classification implements specific complications of internal lengthening devices, not all complications such as regenerative insufficiency have been addressed.

\section{Conclusion}

Due to a low incidence of limb lengthening procedures, there is a lack of valid studies on intramedullary lengthening devices. Direct comparison of specific devices with equivalent treatment protocols and a higher caseload might provide further important information on the efficacy and complications of these devices.

Regarding the lack of control of ISKD ${ }^{\circledR}$ nails in the literature, our results indicate a better control of distraction and patients might have benefited by rigid reaming. Our rate of backtracking of Fitbone ${ }^{\circledast}$ nails was higher than previously reported.

We recommend close follow-up in all procedures in order to facilitate the early detection of specific problems such as backtracking, jamming, implant instability, late or early consolidation, contractures, or neurovascular lesions.

Ethical committee approval was obtained prior to the study. Informed consent was obtained from all individual participants included in the study. 


\section{References}

1. Betz A, Baumgart R, Schweiberer L. First fully implantable intramedullary system for callus distraction--intramedullary nail with programmable drive for leg lengthening and segment displacement. Principles and initial clinical results. Chir Z Für Alle Geb Oper Medizen 1990;61(8):605-609.

2. Cole JD, Justin D, Kasparis T, et al. The intramedullary skeletal kinetic distractor (ISKD): first clinical results of a new intramedullary nail for lengthening of the femur and tibia. Injury 2001;32(Suppl 4): SD129-SD139. DOI: 10.1016/s0020-1383(01)00116-4.

3. Guichet J-M, Deromedis B, Donnan LT, et al. Gradual femoral lengthening with the Albizzia intramedullary nail. J Bone Joint Surg Am 2003;85-A(5):838-848. DOI: 10.2106/00004623-20030500000011.

4. Hankemeier S, Pape H-C, Gosling T, et al. Improved comfort in lower limb lengthening with the intramedullary skeletal kinetic distractor. Princip Prelimin Clin Experien Arch Orthop Trauma Surg 2004;124(2):129-133. DOI: 10.1007/s00402-003-0625-6.

5. Kenawey M, Krettek C, Liodakis $E$, et al. Leg lengthening using intramedullary skeletal kinetic distractor: results of 57 consecutive applications. Injury 2011;42(2):150-155. DOI: 10.1016/ j.injury.2010.06.016.

6. Paley D. PRECICE intramedullary limb lengthening system. Expert Rev Med Devices 2015;12(3):231-249. DOI: 10.1586/17434440.2015.1005604.

7. Bliskunov Al. Intramedullary distraction of the femur (preliminary report). Ortop Travmatol Protez 1983(10):59-62.

8. Thaller PH, Fürmetz J, Wolf $F$, et al. Limb lengthening with fully implantable magnetically actuated mechanical nails (PHENIX( $\left.{ }^{\oplus}\right)$ )preliminary results. Injury 2014;45(1):S60-S65. DOI: 10.1016/j. injury.2013.10.029.

9. Schiedel FM, Vogt B, Tretow HL, et al. How precise is the PRECICE compared to the ISKD in intramedullary limb lengthening? Reliability and safety in 26 procedures. Acta Orthop 2014;85(3):293-298. DOI: 10.3109/17453674.2014.913955.

10. Küçükkaya M, Karakoyun Ö, Sökücü S, et al. Femoral lengthening and deformity correction using the Fitbone motorized lengthening nail. J Orthop Sci Off J Jpn Orthop Assoc 2015;20(1):149-154. DOI: 10.1007/ s00776-014-0659-3.

11. Kirane YM, Fragomen AT, Rozbruch SR. Precision of the PRECICE internal bone lengthening nail. Clin Orthop 2014;472(12):3869-3878. DOI: 10.1007/s11999-014-3575-0.

12. Krieg $A H$, Lenze $U$, Speth $B M$, et al. Intramedullary leg lengthening with a motorized nail. Acta Orthop 2011;82(3):344-350. DOI: 10.3109/17453674.2011.584209.

13. Thaller PH, Degen N, Fürmetz J, et al. Längen-, Achs- und torsionskorrekturen mit distraktionsmarknägeln: erfahrungen mit 5 verschiedenen Systemen. Trauma Berufskrankh 2017(4). DOI: 10.1007/ s10039-017-0336-9.

14. Burghardt RD, Herzenberg JE, Specht SC, et al. Mechanical failure of the intramedullary skeletal kinetic distractor in limb lengthening. J Bone Joint Surg Br 2011;93(5):639-643. DOI: 10.1302/0301620X.93B5.25986.

15. Lee DH, Ryu KJ, Song HR, et al. Complications of the intramedullary skeletal kinetic distractor (ISKD) in distraction osteogenesis. Clin Orthop 2014;472(12):3852-3859. DOI: 10.1007/s11999-014-3547-4.

16. Calder PR, Laubscher M, Goodier WD. The role of the intramedullary implant in limb lengthening. Injury 2017;48(1):S52-S58. DOI: 10.1016/j. injury.2017.04.028.

17. Baumgart $R$, Thaller $P$, Hinterwimmer $S$. A fully implantable, programmable distraction nail (Fitbone) - New perspectives for corrective and reconstructive limb surgery Leung K-S, Taglang G, Schnettler R, et al., ed. Practice of intramedullary locked nails: new developments in techniques and applications. Berlin, Heidelberg: Springer Berlin Heidelberg; 2006. pp. 189-198. DOI: 10.1007/3-54032345-7_19.
18. Thaller PH, Zoffl F, Delhey P. Comparison Between Fully Implantable Monitorozed and Mechanical Distraction Nails - A Matched-Pairs Analysis. 3rd World Congress on External Fixation and 6th Congress of the ASAMI International presented at: Podium Session 37: Bone Reconstruction 6; 2010 Oct 20; Barcelona.

19. Mahboubian S, Seah M, Fragomen AT, et al. Femoral lengthening with lengthening over a nail has fewer complications than intramedullary skeletal kinetic distraction. Clin Orthop Relat Res 2012;470(4): 1221-1231. DOI: 10.1007/s11999-011-2204-4.

20. Laubscher M, Mitchell C, Timms A, et al. Outcomes following femoral lengthening: an initial comparison of the precise intramedullary lengthening nail and the LRS external fixator monorail system. Bone Jt J 2016;98-B(10):1382-1388. DOI: 10.1302/0301-620X.98B10.36643.

21. Horn J, Grimsrud $\varnothing$, Dagsgard $A H$, et al. Femoral lengthening with a motorized intramedullary nail: a matched-pair comparison with external ring fixator lengthening in 30 cases. Acta Orthop 2015;86(2):248-256. DOI: 10.3109/17453674.2014.960647.

22. Burghardt RD, Manzotti $A$, Bhave A, et al. Tibial lengthening over intramedullary nails: a matched case comparison with Ilizarov tibial lengthening. Bone Jt Res 2016;5(1):1-10. DOI: 10.1302/20463758.51.2000577.

23. Paley D, Herzenberg JE, Paremain G, et al. Femoral lengthening over an intramedullary nail. A matched-case comparison with llizarov femoral lengthening. J Bone Joint Surg Am 1997;79(10):1464-1480. DOI: 10.2106/00004623-199710000-00003.

24. Leidinger B, Winkelmann W, RoedI R. Limb lengthening with a fully implantable mechanical distraction intramedullary nail. Z Orthop Ihre Grenzgeb 2006;144(4):419-426. DOI: 10.1055/s-2006-942169.

25. Paley D. Problems, obstacles, and complications of limb lengthening by the Ilizarov technique. Clin Orthop 1990(250):81-104. DOI: 10.1097/00003086-199001000-00011.

26. Lee DH, Kim S, Lee JW, et al. A comparison of the device-related complications of intramedullary lengthening nails using a new classification system. Biomed Res Int 2017;2017:1-9. DOI: 10.1155/2017/8032510.

27. Thaller PH, Wolf F, Kucukkaya M. Surgical techniques for lengthening and deformity correction of the tibia with lengthening nails. Tech Orthop 2014;29(3):150-157. DOI: 10.1097/BTO.0000000000000074.

28. Kucukkaya M, SokucuS, Thaller PH. Surgical techniques for lengthening and deformity correction of the femur with lengthening nails. Tech Orthop 2015;30(3):183-188. DOI: 10.1097/BTO.0000000000000137.

29. Wolf F, Bösl S, Degen N, et al. Impacted steel sleeves for a minimally invasive approach in intramedullary nailing. Injury 2019;50(3):4-10. DOI: 10.1016/j.injury.2019.07.033.

30. Wang K, Edwards E. Intramedullary skeletal kinetic distractor in the treatment of leg length discrepancy-a review of 16 cases and analysis of complications. J Orthop Trauma 2012;26(9):e138-e144. DOI: 10.1097/BOT.0b013e318238b5b1.

31. Accadbled F, Pailhé R, Cavaignac $E$, et al. Bone lengthening using the Fitbone $\left({ }^{\oplus}\right)$ motorized intramedullary nail: the first experience in France. Orthop Traumatol Surg Res OTSR 2016;102(2):217-222. DOI: 10.1016/j.otsr.2015.10.011.

32. Schiedel FM, Pip S, Wacker S, et al. Intramedullary limb lengthening with the intramedullary skeletal kinetic distractor in the lower limb. J Bone Joint Surg Br 2011;93(6):788. DOI: 10.1302/0301620X.93B6.25581.

33. Singh $S$, Lahiri A, Iqbal $M$. The results of limb lengthening by callus distraction using an extending intramedullary nail (Fitbone) in nontraumatic disorders. J Bone Joint Surg Br 2006;88(7):938-942. DOI: 10.1302/0301-620X.88B7.17618.

34. Santolini E, Goumenos SD, Giannoudi M, et al. Femoral and tibial blood supply: a trigger for non-union? Injury 2014;45(11):1665-1673. DOI: 10.1016/j.injury.2014.09.006.

35. Koczewski P, Shadi M. Factors influencing bone regenerate healing in distraction osteogenesis. Ortop Traumatol Rehabil 2013;15(6): 591-599. DOI: 10.5604/15093492.1091515. 